\title{
Shear rheology and phase behaviour of sodium oleate/water mixtures
}

\author{
Filipe E. Antunes ${ }^{\mathrm{b}}$, Luigi Coppola ${ }^{\mathrm{a}, *}$, Danilo Gaudio ${ }^{\mathrm{a}}$, \\ Isabella Nicotera ${ }^{\mathrm{a}}$, Cesare Oliviero ${ }^{\mathrm{a}}$ \\ ${ }^{a}$ Department of Chemistry, University of Calabria, Ponte P. Bucci, Cubo 14/D, 87036 Arcavacata di Rende, Cosenza, Italy \\ ${ }^{\mathrm{b}}$ Department of Chemistry, University of Coimbra, 3004-535 Coimbra, Portugal \\ Received 13 June 2006; received in revised form 11 October 2006; accepted 13 October 2006 \\ Available online 20 October 2006
}

\begin{abstract}
The knowledge of structural and dynamical properties in binary sodium oleate $(\mathrm{NaO}) /$ water mixtures represents a first step for studying new biocompatible structures. In addition, a deeper understanding of their flow behaviour is crucial to control their mechanical properties. The purpose of this investigation was to revisit the binary $\mathrm{NaO}$ /water phase diagram through a combined effort of shear-rheology, NMR-diffusometry, optical polarizing microscopy and differential scanning calorimetry. The preparation of $\mathrm{NaO} /$ water mixtures in this work fitted with the interesting regions of the phase diagrams where most of the new systems will be prepared (i.e. from 0.01 to $30 \mathrm{wt} . \% \mathrm{NaO}$ and within the temperature range $5-70{ }^{\circ} \mathrm{C}$ ).

Steady shear and linear viscoelastic experiments were accurately performed and the relationship between phase morphology and rheological properties was also studied. Regarding the mixtures at low surfactant concentrations, the shear flow showed: (1) a structural evolution into the liquid micellar phase $\left(\mathrm{L}_{1}\right)$ as a function of composition and (2) the presence of a viscous micellar region $\left(\mathrm{L}_{1}^{*}\right)$ induced by temperature and composition changes. $\mathrm{NaO}$ /water mixtures with a liquid-crystalline structure (hexagonal $\mathrm{H}_{1}$ ) were studied by linear shear deformations and paying particular attention to frequency and temperature scans. Viscoelastic spectra of $\mathrm{H}_{1}$ phase presented a gel-like response which, although not expected, indicated the presence of a stiff liquid-crystalline lattice. Finally, a correlation between rheology and microstructural changes was conducted by the "weak-gel" model.
\end{abstract}

(C) 2006 Elsevier B.V. All rights reserved.

Keywords: Sodium oleate; Steady and oscillatory rheology; NMR-diffusometry; Micellar and hexagonal phases

\section{Introduction}

Surfactants play an important role in chemistry with considerable implications for the environment. Above a certain concentration the surfactants undergo a reversible self-aggregation to form micelles. With increasing surfactant concentration the micelles exhibit a special set of structural transitions, transforming from the spherical shape into cylindrical and other shapes [1-3]. Surfactant aqueous solutions usually behave as a Newtonian fluid but in the range of higher frequency or shear rate they exhibit striking viscoelastic behaviour [4]. The viscoelasticity can be seen easily by simple solution swirling and

\footnotetext{
* Corresponding author. Tel.: +390984 492023/492021; fax: +390984 492044 .

E-mail address: lg.coppola@unical.it (L. Coppola).
}

visually observing the recoil movement of air bubbles trapped in the solution after the swirling motion is stopped [5].

Depending of the chemical nature of surfactant, temperature and water concentration, different types of ordered liquidcrystalline phases can also exist (cubic, lamellar and hexagonal phases). Such a fascinating varieties of self-assembled structures lead to occurrence of a wide range of rheological properties under specified shear flows and where the mechanical response is strongly influenced by micro-domain structures. For small strain/flow, the surfactant microstructure is only slightly distorted to exhibit linear viscoelasticity. In this case, shear rheology may assist in the determination of phase diagrams since it is sensitive not only to changes of association structures but also to the variation of both composition and temperature within the ream of existence of a particular mesophase [6].

Biodegradable surface-active molecules are currently receiving a strong attention due to environmental capabilities, however, 
the literature shows a lack of rheological studies on supramolecular structures formed by this kind of amphiphiles. Sodium oleate, $\mathrm{NaO}$, is a carboxylate salt (i.e. the salt form of the unsaturated fatty oleic acid) and an anionic surfactant with amphiphilic capabilities. $\mathrm{NaO}$ exhibits a $120^{\circ}$ rotation in the double link, having an $\mathrm{L}$ form. The presence of a double link affects the molecular packing in the membranes. Sodium oleate has a broad range of applications, from detergency to pharmaceuticals [7].

The control of the self-assembled structures of the $\mathrm{NaO}$ in water is of crucial importance and it should be the first step in the investigations containing this compound (e.g. polymersurfactant biogels). We retain that the system $\mathrm{NaO}$ /water is interesting for industrial applications, constituting a basis for the creation of new types of self-assembled object biosystems $[8,9]$.

The phase diagram of $\mathrm{NaO}$ in water was the subject of two dated studies by Vold [10] and Luzzati et al. [11], respectively. They study the presence of an isotropic liquid region and an hexagonal liquid crystalline phase, lying within an accessible experimental temperature-composition interval. The micellar phase ranged between $\mathrm{cmc}$ and $15 \mathrm{wt}$.\% of surfactant while the hexagonal phase is present at concentration over $15 \mathrm{wt} . \%$ of $\mathrm{NaO}$. In this paper shear rheology (by steady and dynamic experiments) was applied to the liquid and liquid crystalline phases of $\mathrm{NaO}$ /water mixtures at different $\mathrm{NaO}$ compositions and as a function of temperature. The main objectives of this study were (1) characterizing the self-assembled structures of $\mathrm{NaO}$ within the solution and liquid crystalline phase intervals; (2) probing the structural and morphological transitions induced by composition and temperature changes; (3) determining the mechanical properties of these cylindrical surfactant aggregates in order to establish rheological signatures which could be used in comparison with liquid crystalline phases encountered in other aqueous systems.

\section{Experimental}

\subsection{Materials}

Sodium oleate $(\mathrm{NaO})$, or sodium 9-octadecenoate, is an anionic surfactant with $\mathrm{C}_{18}$ hydrophobic chain and a carboxyl headgroup (typical in fatty acids) and a $M_{\mathrm{W}}=304 \mathrm{~g} / \mathrm{mol}$. It was purchased from Nu-Chek Prep, Inc. (Elysian, USA) with high purity $(>99 \%)$ and was used without any further purification. Solutions of different concentrations (within 0.01-30 wt.\% $\mathrm{NaO}$ ) were prepared using double distilled water.

\subsection{Sample preparation and equilibration}

Samples were prepared by weighting the appropriate surfactant and water amounts in glass ampoules containing a small magnetic stirring bar. The samples were flame sealed and mixed in a vortex 708 (ASAL, Italy). After homogenisation at $70^{\circ} \mathrm{C}$, the mixtures were stored for 1 week before measurements. Surfactant solutions were fully transparent and free from foam and air bubbles. Samples were submitted to a polarized microscope to observe the existence of anisotropy or isotropy.

\subsection{Phase diagram determination}

The phase diagram of $\mathrm{NaO} /$ water system was constructed by heating and cooling macroscopic samples in a water bath while observing them through crossed Polaroid plates and by using optical microscopy with a air-flow hot stage where the sample were sealed to prevent water loss. At a lesser extent differential scanning calorimetry was also used, particularly to verify the temperatures at which: (a) the solid surfactant fully dissolved to form either micellar or liquid crystalline phases (Kraft boundary) and (b) the liquid crystalline phase melts in isotropic solutions.

From the general appearance of the ${ }^{2} \mathrm{H}$ NMR spectra it was established whatever a certain sample consisted of a single homogeneous phase or was heterogeneous, i.e. of two or three coexisting phases. Analysis of the ${ }^{2} \mathrm{H}$ NMR spectra as function of the sample composition and temperature enabled us to determine the equilibrium boundary lines of single phases and multiphase regions. The phases were also confirmed by observing the texture in polarizing microscopy.

\subsection{Shear rheology}

Engineering flows are in general three-dimensional and contain both simple shear and rotation-free deformations [12]. Here we limit ourselves to simple shear flow deformations only, and hence do not introduce the full tensioral description of stress and strain. Simple shearing flows are of particular importance as all the rheological measurements made in this contribution are from simple shear deformations. Rheological measurements were conducted using a shear-strain controlled rheometer RFS III (Rheometrics, USA) equipped with a concentric cylinder geometry (inner radius $17 \mathrm{~mm}$, gap $1.06 \mathrm{~mm}$ ). The temperature was controlled by a water circulator heating apparatus $\left( \pm 0.1^{\circ} \mathrm{C}\right)$. To prevent errors due to evaporation, the measuring geometries were surrounded by an evaporation trap.

Rheological responses of $\mathrm{NaO}$ /water mixtures were determined under the kinematics of both steady and oscillatory simple shears. In steady-shear experiments, the apparent viscosity of mixtures was determined from the ratio of measured shear stress $\tau_{21}$ to applied shear rate $\dot{\gamma}$, as a function of shear rate that was varied from 0.02 to $1700 \mathrm{~s}^{-1}$. Steady states were previously checked by transient experiments (step-rate test). For concentrations lower than $10 \mathrm{wt} . \% \mathrm{NaO}$ it was observed that $10 \mathrm{~s}$ was a sufficient scanning time to ensure the steady state condition. For concentrated solutions and liquid crystalline mixtures, steady state viscosity was determined from long-time shear stresses.

It is generally accepted that microstructure within a structured fluids must be distorted when it is deformed at shear rate that exceeds the reciprocal of the characteristic time scale for microscopic motion. Hence, steady shear is not ideally suited if one wants to probe the rheological characteristics of an unperturbed mixture. In oscillatory shear experiments, the specimen is subjected to a very small oscillatory strain, such that its structure remains intact. Small amplitude oscillatory shear strains 
$\gamma_{21}$ were imposed on the samples at a fixed frequency $\omega$ and a maximum strain amplitude $\gamma_{0} \ll 1$ :

$\gamma_{21}(t)=\gamma_{0} \sin \omega t$

The angular frequency $\omega=2 \pi f$, in $\mathrm{rad} / \mathrm{s}$, is defined by the number of cycles per time given by the frequency $f, \mathrm{~Hz}$. Within the linear viscoelastic region, the resulting shear stress, $\tau_{21}$, is also sinusoidal and can be decomposed into an in-phase and out-of-phase component:

$\tau_{21}(t)=G^{\prime} \gamma_{0} \sin \omega t+G^{\prime \prime} \gamma_{0} \cos \omega t$

The stress component in-phase with the deformation defines the elastic (or storage) modulus $G^{\prime}$ and is related to the elastic energy stored in the system on deformation. The component outof-phase with the stain gives the viscous (or loss) modulus $G^{\prime \prime}$ which is linked to the viscous dissipation of energy in the system. The moduli $G^{\prime}$ and $G^{\prime \prime}$ are function only of the oscillatory frequency $\omega$ for low deformation confined within the linear region. To evaluate the characteristics of a viscoelastic material, it is customary to examine the frequency spectrum showing $G^{\prime}$ and $G^{\prime \prime}$ as function of $\omega$. The relative magnitudes and shapes of $G^{\prime}(\omega)$ and $G^{\prime \prime}(\omega)$ curves indicate the type and extend of microstructure present. In this study $\omega$ was varied between 0.1 and $10^{2} \mathrm{rad} / \mathrm{s}$.

Although elastic modulus $G^{\prime}(\omega)$ is an important indicator of the structuring degree in a colloidal system, an other useful parameter which is dimensionless and convey no physical magnitude is the loss tangent, $\tan \delta(\omega) . \delta(\omega)$ is the shift-phase angle between shear stress and strain. The loss tangent is a measure of the ratio of the energy lost to energy stored in a cyclic deformation and defined as:

$\tan \delta(\omega)=\frac{G^{\prime \prime}(\omega)}{G^{\prime}(\omega)}$

It has been shown that the linear dynamic rheology of surfactant systems could follow, in first approximation, a simple Maxwell model with a single relaxation time. According to this, $G^{\prime}$ and $G^{\prime \prime}$ are linked each other in a complex modulus $G^{*}(\omega)$ which is given by [13]:

$G^{*}(\omega)=G^{\prime}(\omega)+i G^{\prime \prime}(\omega)=\frac{i \omega \eta_{0}}{1+i \omega \lambda}$

The relaxation time $\lambda$ is the characteristic time at which the structured fluid relaxes back to the equilibrium configuration when perturbed by a shear oscillation. This time, also called the "terminal relaxation time", separates two regimes:

(i) For $\omega \lambda \ll 1$, the system can be considered as a viscous fluid with viscosity $\eta_{0}$. This regime is called "terminal flow" behaviour of a Maxwell fluid where $G^{\prime \prime}(\omega)=\omega \eta_{0}$, $G^{\prime}(\omega)=\omega^{2} \lambda \eta_{0}$ and $\tan \delta>1$.

(ii) For $\omega \lambda \gg 1$, the system exhibit elasticity. In this regime, the rubber-like zone, the storage modulus is independent of the frequency: $G_{0}=\eta_{0} / \lambda\left(G_{0}\right.$ is the plateau value of $G^{\prime}$ at high frequency), and $G^{\prime \prime}$ and $\tan \delta \rightarrow 0$.

In a recent paper, Mackley et al. [14] pointed out that a complete rheological characterization of colloidal systems could be better conducted either by a generalized Maxwell model or by a strain-dependent exponential dumping function.

\section{5. ${ }^{2} H$ NMR spectroscopy}

${ }^{2} \mathrm{H}-\mathrm{NMR}$ spectra were performed in a Bruker NMR spectrometer AVANCE 300 with wide-band probe $(5 \mathrm{~mm})$, operating at $46 \mathrm{MHz}$ on deuterium. An airflow regulator controls the temperature in the measuring probe $\left( \pm 0.3^{\circ} \mathrm{C}\right)$. The quadrupolar echo technique was used for data acquisition. Before the Fourier transformation, 256 transients at a repetition rate of $0.5 \mathrm{~s}$ were averaged.

\section{6. ${ }^{1}$ H NMR diffusometry}

Water and surfactant self-diffusion coefficients $\left(D_{\mathrm{w}}\right.$ and $\left.D_{\mathrm{s}}\right)$ were determined in a Bruker NMR spectrometer AVANCE 300 Wide Bore working at $300 \mathrm{MHz}$ on ${ }^{1} \mathrm{H}$. The employed probe was a Diff30 Z-diffusion $30 \mathrm{G} / \mathrm{cm} /$ A multinuclear. All samples were hermetically sealed in $5 \mathrm{~mm}$ Pyrex tubes. The sealed sample tubes were first heated in an oven to $353 \mathrm{~K}$ for homogenization and then slowly cooled to room temperature. The phase sequence was checked by crossed polarized lens with increasing the temperature. The samples were then loaded into the NMR probe at the desired temperature. ${ }^{1} \mathrm{H}$ self-diffusion coefficients and the correspondent spectra were taken at different temperatures. The temperature was changed at slow rate $\left(1 \mathrm{~K} \mathrm{~min}^{-1}\right)$ and samples were equilibrated at each temperature for approximately $30 \mathrm{~min}$.

The diffusion experiments were performed using the "pulsed field gradient spin-echo" (PGSE) sequence described by Stejskal and Tanner [15]. Diffusion coefficients were obtained using:

$I(\delta, \Delta, g)=I_{0} \exp \left[-\gamma^{2} g^{2} \delta^{2}\left(\Delta-\frac{\delta}{3}\right) D\right]$

where $I(\gamma, \Delta, g)$ and $I_{0}$ are the echo intensities in presence of gradient pulses of strength $\mathrm{g}$ and in absence of gradient pulses, respectively. The length of gradient pulse is $\delta, \Delta$ is the distance between leading edges of the gradient pulses, and $\gamma$ is the gyromagnetic ratio (for protons $\gamma=26.725 \times 10^{7} \mathrm{rad} \mathrm{T}^{-1} \mathrm{~s}^{-1}$ ). The value of $\Delta$ and $\delta$ used for water self-diffusion measurements were 140 and $0.5 \mathrm{~ms}$, respectively, while $\delta$ was $5 \mathrm{~ms}$ for surfactant self-diffusion measurements. In the experiment $\mathrm{g}$ was incremented from 0.20 to $3 \mathrm{~T} \mathrm{~m}^{-1}$ and from 1 to $9 \mathrm{Tm}^{-1}$ for water and surfactant, respectively. The pure water self-diffusion was measured to $2.29 \times 10^{-9} \pm 0.04 \mathrm{~m}^{2} \mathrm{~s}^{-1}$ at $25^{\circ} \mathrm{C}$. The experimental data were analyzed by non-linear least-square regression of Eq. (5) using a Bruker-made algorithm. The uncertainty in self-diffusion measurements was $\sim 2 \%$.

\subsection{Differential scanning calorimetry}

DSC studies were performed on the samples using a Setaram DSC-131 instrument, which used indium to calibrate temperature and energy scales. Samples $(20-30 \mathrm{mg}$ ) were sealed in aluminium-cells and heated to the initial temperature. Thermograms were digitised by an IBM computer, which allowed the 
determination of the phase transition temperatures with the associated heat changes by the use of the commercial software Origin 7.5 (OriginLab, MA USA).

\section{Results and discussion}

\subsection{Phase diagram}

Although this study concerns mainly with rheological properties of $\mathrm{NaO}$ /water mixtures, in this section we report for opportunity the description of the partial phase diagram in water. It was carefully reinvestigated by means of complementary physicochemical techniques like optical polarizing microscopy, differential scanning calorimetry (DSC), ${ }^{2} \mathrm{H}$ NMR spectroscopy, NMR-diffusometry and shear rheology). The partial phase diagram is sketched in Fig. 1 and refers to mixtures in the composition range $0.01 \mathrm{wt} . \%<c<30 \mathrm{wt} . \% \mathrm{NaO}$ and the temperature interval of $5-70{ }^{\circ} \mathrm{C}$. The hydrolysis degree of $\mathrm{NaO} /$ water mixtures, leading to oleic acid and which could modify the aggregate structure, has been estimated by $\mathrm{pH}$ measurements. The proportion of oleic acid which is formed remained negligible in the whole concentration range investigated. In this study, samples exhibited $\mathrm{pH}$ within 8.3-8.4, independently of concentrations. These findings agree with previously reported studies [16].

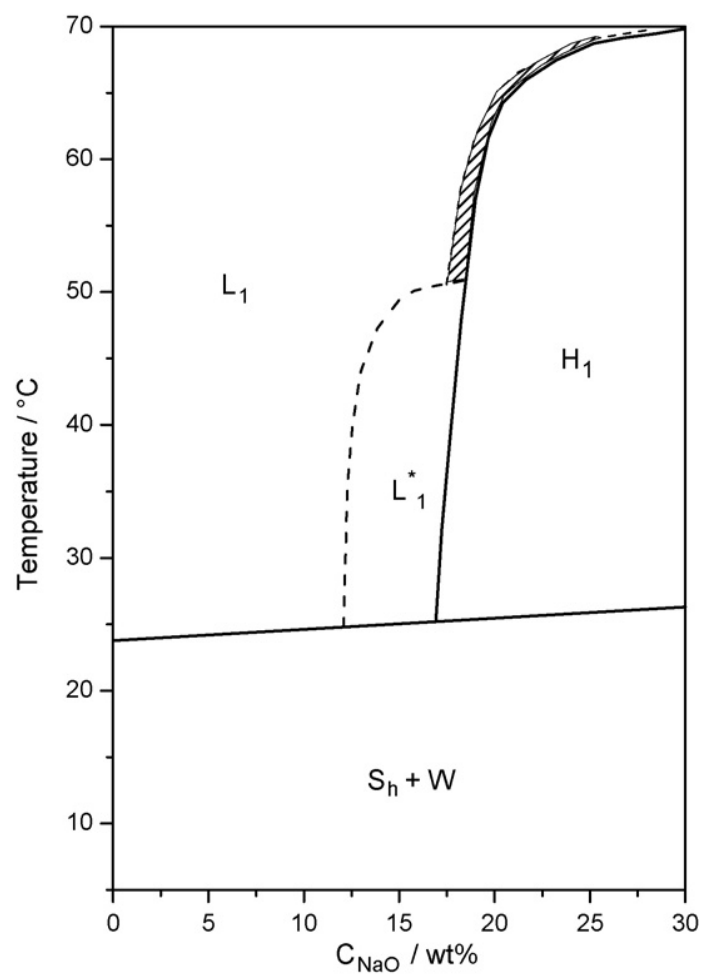

Fig. 1. Partial temperature-composition phase diagram for the sodium oleate $(\mathrm{NaO}) /$ water system. The micellar solution phase is denoted by $\mathrm{L}_{1}$ while $\mathrm{H}_{1}$ denotes the hexagonal liquid crystalline phase, according to the Tiddy's notation [3]. $\mathrm{L}_{1}^{*}$ represents a viscous micellar solution which becomes birefringent under weak shearing. $\left(S_{\mathrm{h}}+W\right)$ denotes a region with hydrated surfactant crystals plus water; the dashed area is a narrow $\left(\mathrm{L}_{1}+\mathrm{H}_{1}\right)$ region. The resolution of the phase boundary is better than $\pm 2 \%$.
Within the temperature interval $5-27^{\circ} \mathrm{C}$, the phase diagram presents a cloudy two-phase region consisting of hydratedcrystalline surfactant and pure water $\left(S_{\mathrm{h}}+W\right)$. The Krafft discontinuity is slightly changed with increasing the surfactant composition. This result is in agreement with studies by Vold [10], where this discontinuity was identified as the temperature at which the last trace of opaque solid disappears by heating.

Above $27^{\circ} \mathrm{C}$ and for the whole surfactant concentration analyzed, the phase diagram is characterized by a clear isotropic non-viscous micellar phase $\left(\mathrm{L}_{1}\right)$ and a liquid crystal hexagonal phase $\left(\mathrm{H}_{1}\right)$. The presence of $\mathrm{H}_{1}$ phase was clearly deducted by the presence of typical "fanlike" textures in optical polarizing micrographs. On this subject, Borné [8] reported the occurrence of $\mathrm{D}_{2} \mathrm{O}-\mathrm{NMR}$ Pake-doublets which is characteristic of a direct hexagonal self-assembling with a homogeneous orientation of directors.

Along the isothermal path at $35^{\circ} \mathrm{C}$, the $\mathrm{NaO}$ /water mixtures present the following phase sequence: isotropic micellar solution $\left(\mathrm{L}_{1}\right) \rightarrow$ hexagonal phase $\left(\mathrm{H}_{1}\right)$. However, while the micellar solutions for $c<12 \mathrm{wt} . \% \mathrm{NaO}$ are not birefringent and do not exhibit streaming birefringence, within the concentration range $12-17 \mathrm{wt} \%$ the solution mixtures showed a birefringence induced by shear. This zone of micellar phase was accurately investigated and was indicated as $\mathrm{L}_{1}^{*}$ to mark the difference on the physicochemical properties with respect to the dilute one. At concentrations higher than $18 \mathrm{wt} . \%, \mathrm{NaO}$ forms a direct hexagonal liquid-crystalline phase. The upper phase boundaries of $\mathrm{H}_{1}$ depend on the concentration of surfactant and at $30 \mathrm{wt} . \%$ $\mathrm{NaO}$ the phase transition (corresponding to the melting of $\mathrm{H}_{1}$ ) was observed at ca. $70^{\circ} \mathrm{C}$ by using recorded DSC thermograms. Finally, we were not able to prepare homogeneous bulk mixtures for $c>30 \mathrm{wt} . \% \mathrm{NaO}$. This region may be regarded as very viscous two phase area which appeared optically nonuniform.

\subsection{Micellar phase}

To characterize the $\mathrm{L}_{1}$-phase we first performed steady-state rheology. The mixtures were submitted to steady rate-sweep tests and the corresponding long time stresses were measured. Two types of steady flows were observed. Dilute $\mathrm{L}_{1}$ micellar mixtures ( $2 \mathrm{wt} . \%<c<10 \mathrm{wt} \% \mathrm{NaO}$ ) showed a constant viscosity and are referred by Newtonian liquids. At higher concentrations the viscosity becomes a shear-rate dependent function, exhibiting increasingly pronounced shear thinning flow behaviour.

Steady shear tests of $\mathrm{L}_{1}$ micellar solutions resulted also in several temperature-dependent flow curves. The Newtonian viscosity was observed to decrease with increasing the temperature following an Arrhenius-type decay $\left(\ln \eta_{\mathrm{N}} \propto-T\right)$ up to $60^{\circ} \mathrm{C}$. Fig. 2 is representative of flow curves for the sample with the composition 2 wt. $\% \mathrm{NaO}$ as a function of temperature. All viscosity trends in the diluted micellar region $(c<10 \mathrm{wt} . \%)$ are Newtonian and the viscosity values are particularly low (ca. $10^{-3} \mathrm{~Pa}$ s). Such a flow curves were measured by a double-wall Couette that allowed us to reach a great accuracy for low- 


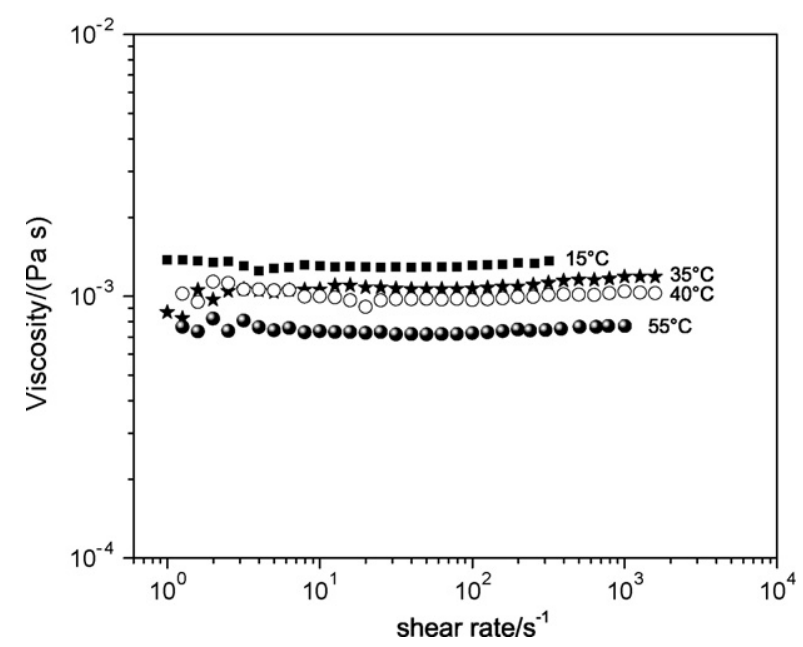

Fig. 2. Newtonian viscosity vs. shear rate $\left(\mathrm{s}^{-1}\right)$ for the $2 \mathrm{wt} . \% \mathrm{NaO}$ mixture, at different temperatures. Rate-sweep experiments performed with a scanning time of $10 \mathrm{~s}$.

viscosity values. The present rheological behaviour is a signature of the presence of a spherical micellar microstructures and indicates the absence of structure-forming interactions between the aggregates. Borné observed the presence of spherical micelles in a $5 \mathrm{wt} . \% \mathrm{NaO}$ mixture by means of cryo-TEM images [8].

Fig. 3 is representative of the flow behaviour for the concentrated micellar zone of this system $(c>10 \mathrm{wt} . \%)$. The mixture with $c=12$ wt. $\% \mathrm{NaO}$ exhibits a plateau in the viscosity curve at shear rates lower than $10^{2} \mathrm{~s}^{-1}$. However, the viscosity decreases at higher shear rates showing a shear-thinning behaviour that appears more pronounced for the sample at $c=15 \mathrm{wt} . \% \mathrm{NaO}$. Steady shear data of the mixture $c=15 \mathrm{wt} . \% \mathrm{NaO}$ were fit by the simplified cross-equation [17] to obtain the zero-shear viscosity, $\eta_{0}$, and an estimation of the shear relaxation exponent $m$ :

$\eta(\dot{\gamma})=\frac{\eta_{0}}{1+(\lambda \dot{\gamma})^{m}}$

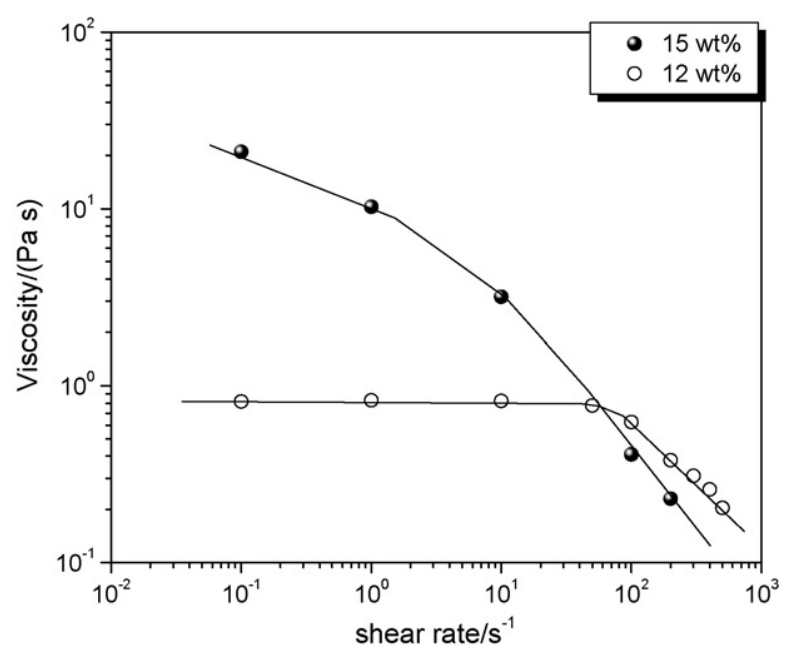

Fig. 3. Steady-state apparent viscosity vs. shear rate $\left(\mathrm{s}^{-1}\right)$ for two selected micellar $\mathrm{L}_{1}$-mixtures: 12 and $15 \mathrm{wt} . \% \mathrm{NaO}$ at $30^{\circ} \mathrm{C}$. Step-rate experiments conducted using a long time scanning analysis. The solid lines represent the best fitting of the simplified Cross model.
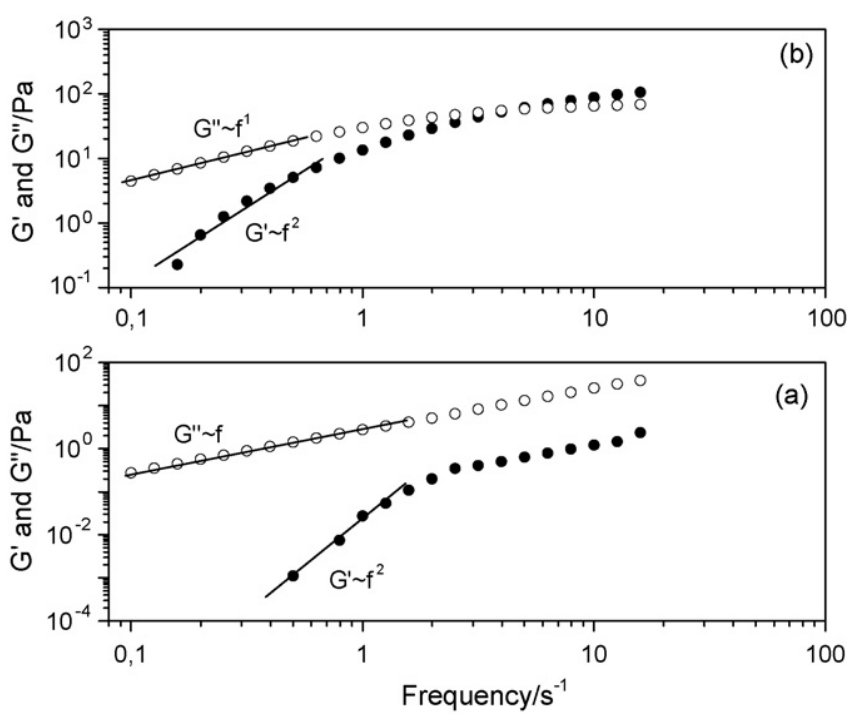

Fig. 4. Linear viscoelastic spectra for two NaO/water mixtures: (a) is 2 wt.\% $\mathrm{NaO}$ and (b) $15 \mathrm{wt} . \% \mathrm{NaO}$ at $30^{\circ} \mathrm{C}$. Frequency evolution of storage modulus $G^{\prime}$ (filled symbols) and loss modulus $G^{\prime \prime}$ (open symbols) obtained by a frequencysweep test at $2 \%$ strain with a scanning time of $5 \mathrm{~s}$.

The exponent $m$ was found to be ca. 0.90 and close to the theoretical exponent for plastic materials $(m=1)$, suggesting a sensitive shear-induced modification or alignment. On the other hand, the slope of the power-law region of flow curves for some dilute worm-like micelles (i.e. long and flexible cylinders) of cationic surfactants/water systems [18,19] was found to be ca. 0.89 . In comparison to this result, we may conclude that shear thinning behaviour observed for $c>10 \mathrm{wt} . \% \mathrm{NaO}$ is due to the anisometric nature of cylindrical aggregates that orient themselves in high shear-rate regime.

Linear viscoelastic responses of $\mathrm{NaO}$ /water of micellar mixtures were obtained by dynamic small strain experiments consisting of frequency and temperature scans. The linear viscoelastic conditions were defined by preliminary strain-sweep tests. Fig. 4 is representative of linear frequency spectra for both diluted ( $2 \mathrm{wt} . \%)$ and concentrated (15 wt.\%) micellar mixtures of $\mathrm{NaO} /$ water system at $30^{\circ} \mathrm{C}$. Several types of information can be extracted from these rheological data. In both mixtures here reported, oscillatory measurements show that viscoelasticty is dominated by a viscous part corresponding to the terminal flow behaviour of a Maxwell fluid. As the frequency increases, the storage modulus $G^{\prime}$ increases faster than the loss modulus $G^{\prime \prime}$ and the curves intersect at a characteristic frequency, $\omega_{\mathrm{c}}$, that is dependent on surfactant concentration. At the crossover point the moduli functions define the longest relaxation time by the simple relation $\lambda \omega c=1$. Dilute micellar solutions have a relaxation time so short (few milliseconds) that it would be necessary to measure it at very high frequency and it can be cumbersome in a classical rheometer (Fig. 4a). As the surfactant concentration increases, it is observed the presence on the frequency spectrum of a certain elasticity inducing the crossover point to fall in the investigated frequency window (Fig. 4b). Micellar dynamics for the $15 \mathrm{wt} . \%$ surfactant solution at $30^{\circ} \mathrm{C}$ are dominated by a relaxation time, $\lambda$, of the order of ca. $200 \mathrm{~ms}$ and a plateau 
modulus, $G_{0}$, of ca. $80 \mathrm{~Pa}$. This value is much lower of that measured in the same dynamic conditions for worm-like micellar solution of cetyltrimethylammonium tosylate (CTAT)/water system (i.e. $G_{0} \approx 400 \mathrm{~Pa}$ and $c=23 \mathrm{wt} . \% \mathrm{CTAT}$ ) at $30^{\circ} \mathrm{C}$ [20]. In the light of these facts, one can distinguish two main structural regimes within the composition range of the micellar solution. First, at low surfactant concentrations $(<10 \mathrm{wt} . \%)$ the solutions are Maxwell fluids with structural relaxation time of few $\mathrm{ms}$ in agreement with the dynamics of dilute spherical aggregates. Second, by increasing of the surfactant concentration, from $c>12 \mathrm{wt} . \%$ and up to the borders of the hexagonal phase, $\mathrm{NaO}$ forms cylindrical aggregates. The liquid-like character of the frequency spectra and low values of plateau moduli measured at $30^{\circ} \mathrm{C}$ indicate the absence of a transient entangled phase like that observed for worm-like mixtures of cationic surfactants.

Linear viscoelastic measurements offer a potent nondestructive method for investigating structural transitions induced by temperature changes in colloidal systems by means of so-called time-cure experiments. These experiments consist of continuously monitoring the evolution of the viscolestic moduli at constant frequency during a temperature ramp, usually carried out at constant heating rate.

Fig. 5 shows temperature-sweep test that we performed on the sample $c=15 \mathrm{wt} . \%$ of $\mathrm{NaO}$. The evolution of material function $\left(G^{\prime}, G^{\prime \prime}\right.$ and $\left.\tan \delta\right)$ of Fig. 4 reveals three different rheological regions as the temperature was raised from 10 to $50^{\circ} \mathrm{C}$ :

- Region I, from 10 to $23^{\circ} \mathrm{C}$, reports that both mechanical moduli $\left(G^{\prime}>G^{\prime \prime}\right)$ decrease with a comparable slope and cross over at ca. $\mathrm{T}=23^{\circ} \mathrm{C}$. This crossover point can be considered as an appropriate measure of onset of phase transition from the solid-like phase $\left(S_{\mathrm{w}}+W\right)$ to isotropic liquid solution $\left(\mathrm{L}_{1}\right)$. Indeed, this temperature is in agreement with the melting of the $\mathrm{NaO}$ surfactants (Krafft point) revealed by appropriate DSC thermograms.

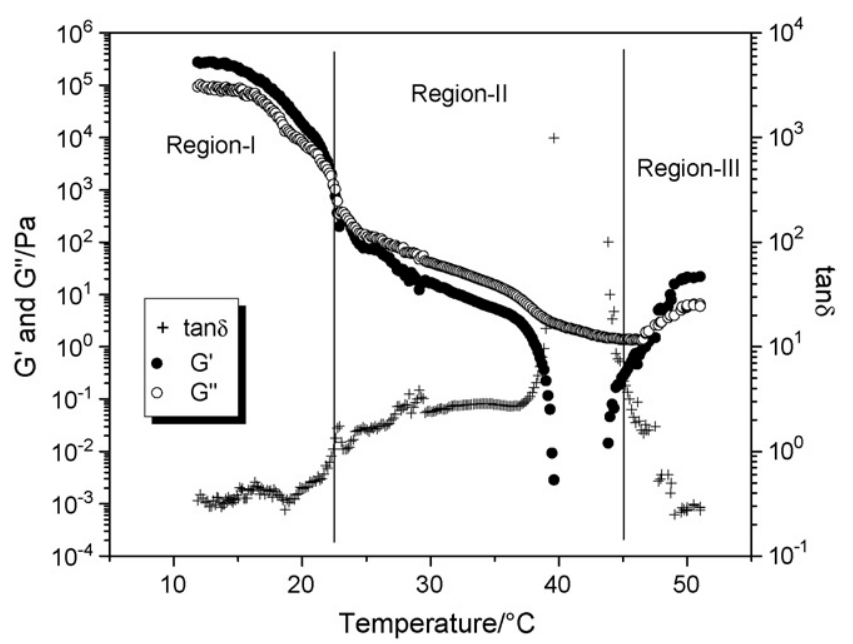

Fig. 5. Linear viscoelastic thermogram for $15 \mathrm{wt} . \% \mathrm{NaO}$ mixture. Temperature scan of storage modulus $G^{\prime}$ (filled symbols), loss modulus $G^{\prime \prime}$ (open symbols) and loss tangent $G^{\prime \prime} / G^{\prime}$ (crosses) obtained by a time-cure test at $2 \%$ and $f=1 \mathrm{~Hz}$. Heating rate of the experiment was of $0.2^{\circ} \mathrm{C} / \mathrm{m}$ and the equilibration time of $2 \mathrm{~s}$.
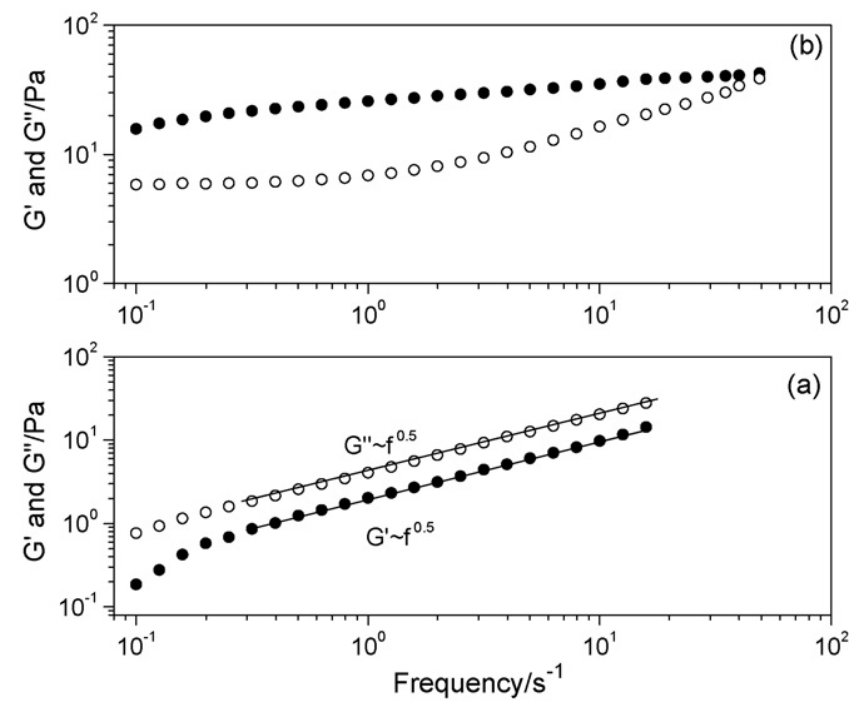

Fig. 6. Linear viscoelastic spectra for $15 \mathrm{wt} . \% \mathrm{NaO} /$ water mixture at two selected temperature: (a) $T=37^{\circ} \mathrm{C}$ and (b) $T=50^{\circ} \mathrm{C}$. Frequency evolution of storage modulus $G^{\prime}$ (filled symbols) and loss modulus $G^{\prime \prime}$ (open symbols) obtained by a frequency-sweep test at $2 \%$ strain with a scanning time of $5 \mathrm{~s}$.

- Region II, which appears at temperatures higher than $27^{\circ} \mathrm{C}$, corresponds to the temperature evolution of mechanical moduli within the $\mathrm{L}_{1}$ phase. After an initial decrease of $G^{\prime}$ and $G^{\prime \prime}$ within increasing the temperature following a quite similar rate, $G^{\prime}$ abruptly disappears at the temperature of $40^{\circ} \mathrm{C}$ whereas $G^{\prime \prime}$ continues on a slow decrease.

- Region III, which starts when temperature is of ca. 45, define an unusual rheological response of this micellar mixture. By a further temperature increase: $G^{\prime}$ abruptly reappears, then it crosses over with $G^{\prime \prime}$ (at ca. $47^{\circ} \mathrm{C}$ ) and finally shows an evident and unexpected gel-like evolution as the temperature increased.

The analysis we described is also confirmed by frequency sweep tests performed at different temperatures. Fig. 6 presents frequency spectra for $15 \mathrm{wt} . \%$ mixture at 37 and $50^{\circ} \mathrm{C}$. At $37^{\circ} \mathrm{C}$ the linear viscolestic data show a liquid-like response $\left(G^{\prime \prime}>G^{\prime}\right)$ that, however, does not longer follow the behaviour of a Maxwell fluid. For frequencies higher than $0.2 \mathrm{~s}^{-1}$ the elastic and viscous modulus scales as: $G^{\prime}(\omega) \sim G^{\prime \prime}(\omega) \sim \omega^{0.5}$. At $50^{\circ} \mathrm{C}$ the frequency dependence of mechanical moduli shows an tremendous different frequency behaviour. Within the frequency interval $0.1-10 \mathrm{~s}^{-1}, G^{\prime}$ is higher than $G^{\prime \prime}$ and both are almost independent of frequency following an evolution which is typical of gel-like responses of polymer solutions. At frequencies higher than $10 \mathrm{~s}^{-1}$, both storage and loss moduli increase with a steeper monotonic increase of $G^{\prime \prime}$, and a crossover is observed in the limit of the frequency window.

NMR-diffusometry has proved to be a very convenient tool to measure the lateral diffusion of each component separately in multi-component isotropic solutions formed in surfactant systems [4]. The monitoring time in the measurement of selfdiffusion coefficients is of the order on ca. $100 \mathrm{~ms}$ and during this time span all measured self-diffusion coefficients correspond to displacements of macroscopic distance several order of magni- 
tude larger than any structural element in the solution. As shown by extensive studies realized by our research group [21-23], the knowledge of individual component self-diffusion behaviour in multi-component solutions provides a unique source of information with regard to the solution structure. For example, in a normal micellar solution, the diffusion coefficient of the continuous phase, water $D_{\mathrm{w}}$, is very rapid and larger than that of the aggregated surfactant molecules, $D_{\mathrm{s}}$, by one to two order of magnitude. On the other hand, in a reverse micellar solution with oil as continuous phase the self-diffusion coefficient of oil is rapid while $D_{\mathrm{s}}$ and $D_{\mathrm{w}}$ are slow. However, if the system forms only molecular solution, the self-diffusion coefficients of all measured components are high and, under ideal condition, the component self-diffusion coefficients are almost identical to their respective components in the neat state.

Fig. 7a reports the surfactant self-diffusion measured within the isotropic solution as a function of the temperature. In the dilute region, $D_{\mathrm{s}}$ shows a slight decrease with increasing the surfactant concentration. Above $c>8 \mathrm{wt}$. \% the surfactant selfdiffusion coefficients evidence a abrupt jump and reduce their
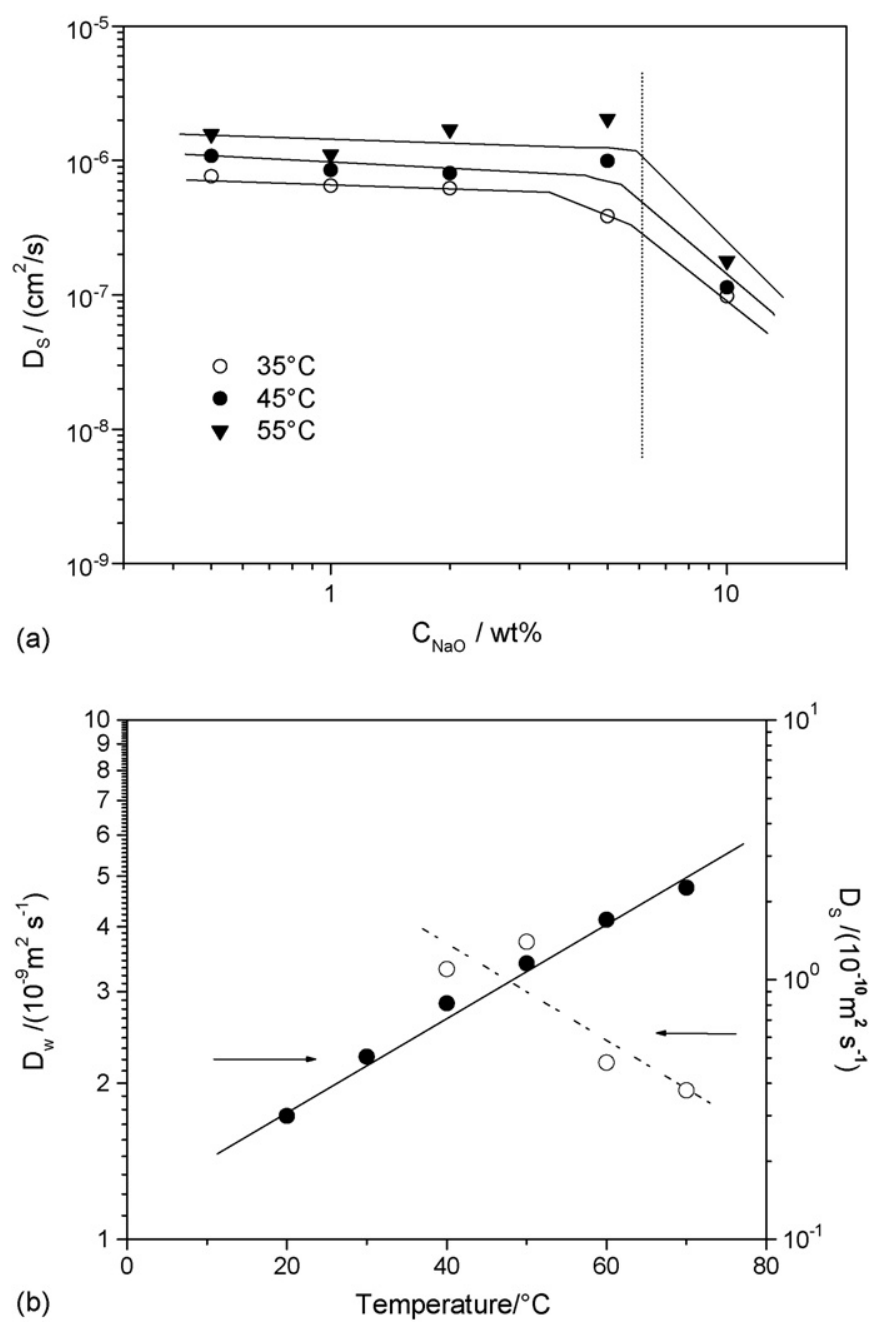

Fig. 7. (a) Surfactant self-diffusion vs. the surfactant composition (dilute micellar phase $\mathrm{L}_{1}$ ) as a function of the temperature. (b) Water and surfactant self-diffusion as a function of the temperature for the $15 \mathrm{wt} . \% \mathrm{NaO}$ mixture. value of a factor 4 . As observed for the flow curve profiles, the self-diffusion data confirm that, in a small composition interval centred on $c=8 \mathrm{wt} . \%$, a change of the micellar structure from a spherical shape to cylindrical aggregates. In addition, it is worthy to note that this structural change takes place at the same composition considering a temperature interval from 30 to $55^{\circ} \mathrm{C}$. Apart from this dilute micellar region $(c<12 \mathrm{wt} . \%)$ we were not longer able to realize accurate PGSE-NMR measurements since micelles presented short surfactant relaxation times (i.e. $T_{2} \cong 15 \mathrm{~ms}$ ). This is an other evidence of the presence of anisometric micelles characterized by sensitively high static dipole-dipole interactions and slow motions.

To better investigate the behaviour observed on the timecure test regarding the $15 \mathrm{wt} . \%$ mixture, we performed several experiments of NMR-diffusometry in order to follow the translational mobility of water and surfactant with increasing the temperature (from 20 up to $70^{\circ} \mathrm{C}$ ). Fig. $7 \mathrm{~b}$ shows a log-lin plot of water and surfactant self-diffusion coefficients versus the temperature. The water self-diffusion coefficients increase linearly showing an Arrhenius trend $\left(\ln D_{\mathrm{w}} \sim T\right)$ which is usually observed in micellar solutions where no structural changes take place increasing the temperature [23]. On the other hand, the surfactant self-diffusion coefficients $\left(T>40^{\circ} \mathrm{C}\right)$ decrease with increasing the temperature following a linear decay which is, however, in agreement with the trend of $G^{\prime}$ observed on the time-cure scan. The temperature behaviour here reported for the $15 \mathrm{wt} . \%$ mixture cannot be easy explained in terms of structural micellar changes. On the other hand, no birefringence was observed despite the unusual rheological and dynamic parameters above described. At this point, what we still have to do is to remark this unusual behaviour and postpone a better analysis to next investigations concerning mainly the effect of the temperature on micellar microstructures.

\subsection{Hexagonal liquid crystalline phase}

Spontaneous liquid crystalline self-assembling was found in mixtures with composition $c>15 \mathrm{wt} . \% \mathrm{NaO}$ and $T>27^{\circ} \mathrm{C}$. This region was identified as a direct hexagonal phase by means of optical microscopy showing a temperature-composition phase boundary as shown in Fig. $1 . \mathrm{H}_{1}$-phase consists of long cylindrical micelles lying parallel to each other and arranged in a hexagonal lattice with a typical distance between micelles of a few nanometers. Hydrocarbon portion of $\mathrm{NaO}$ molecules are direct to the centre of cylinders and hydrophilic portions are located on the external surface of cylinders. From a macroscopic point of view this phase looked like quasi-transparent gels exhibiting high mechanical consistency. It should take in account that as in lyotropic lamellar mesophases, the spontaneous microstructure is polycrystalline with hexagonal mono-domains separated by intervening grain boundaries.

Fig. 8 reports the linear viscoelasticty for two mixtures $(c=18$ and $25 \mathrm{wt} . \% \mathrm{NaO}$ ) at $30^{\circ} \mathrm{C}$ in the frequency range $0.1-15 \mathrm{~Hz}$. Frequency spectra evidenced a viscoelastic response, which is typical of a gel-like material [24]. Storage modulus, $G^{\prime}$, and loss modulus, $G^{\prime \prime}$, are only slightly dependent on the frequency over 

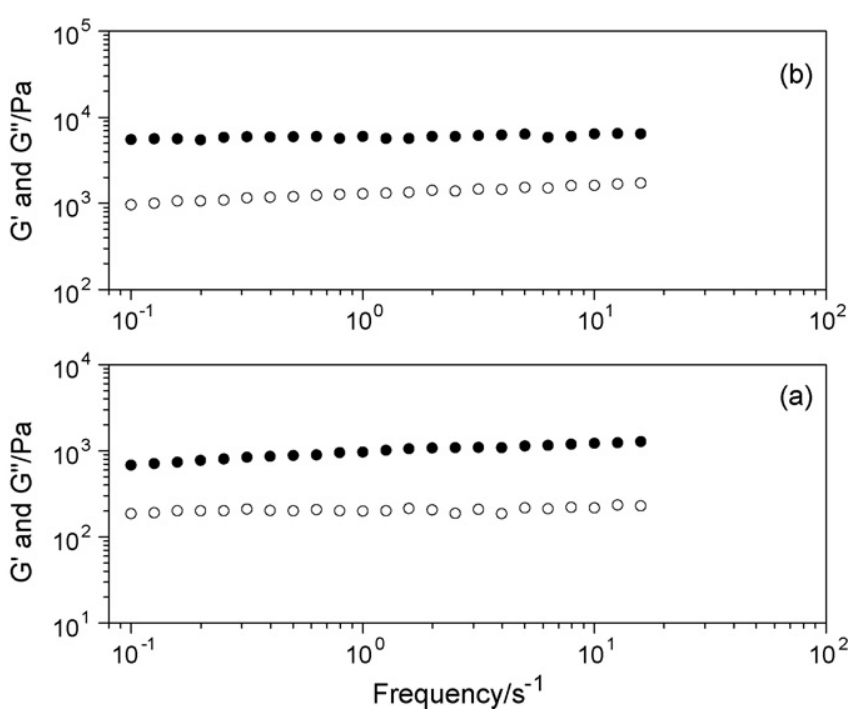

Fig. 8. Linear viscoelastic spectra for two $\mathrm{NaO} /$ water mixtures: (a) is $18 \mathrm{wt} . \%$ $\mathrm{NaO}$ and (b) $25 \mathrm{wt} . \% \mathrm{NaO}$ at $30^{\circ} \mathrm{C}$. Frequency evolution of storage modulus $G^{\prime}$ (filled symbols) and loss modulus $G^{\prime \prime}$ (open symbols) obtained by a frequencysweep test at $2 \%$ strain with a scanning time of $5 \mathrm{~s}$.

the frequency range accessed and scales as [25]:

$G^{\prime}(\omega) \approx G^{\prime \prime}(\omega) \approx \omega^{n}$

$\tan \delta=\tan \left(\frac{n \pi}{2}\right)$

As observed by Winter and co-worker [25] this power law behaviour indicates that the gel structure is self-similar over a wide range of length scale, i.e. a fractal structure characterized by the relaxation exponent $\mathrm{n}$ that has been reported to vary in the range $0<n<1$. The relaxation exponent $n$ describes not only the statistical properties of the gel but could be correlated to the equilibrium microscopic structure of the material. For what concerns the viscoleasticity of this hexagonal phase it must be pointed out that the observed gel response $\left(G^{\prime} / G^{\prime \prime}>10\right.$ over all frequency range explored) is not common in this type of twodimensional lyotropic microstructure.

Only a small number of papers on the rheology of hexagonal mesophases have been published. Recent studies Refs. [26,27] have reported that frequency scans for a hexagonal phase all follow the same general trend that is: $G^{\prime \prime}$ exceeds $G^{\prime}$ at low frequency; at higher frequencies $G^{\prime}$ and $G^{\prime \prime}$ intersect at a characteristic frequency whose reciprocal corresponds to the main relaxation time (of the order of seconds) and above this cross over frequency the phase has a predominantly elastic response. This behaviour corresponds to the transition to the flow region expected by the Maxwell model (Eq. (4)) and means that $\mathrm{H}_{1}$ phase behaves as a viscoelastic fluid capable to flow under long-time applied strains. The absence of the "terminal flow" region for the $\mathrm{NaO}$ system is a signature of a highly ordered $\mathrm{H}_{1}$ phase whose lyotropic domains form a permanent network able to sustain long-time stimuli. Therefore, dynamics of $\mathrm{H}_{1}$-phase are characterized by a spectrum of relaxation times that convolve relaxation process on both fast and slow time scales [28]. We will discuss in the next section the relationship between the gel-

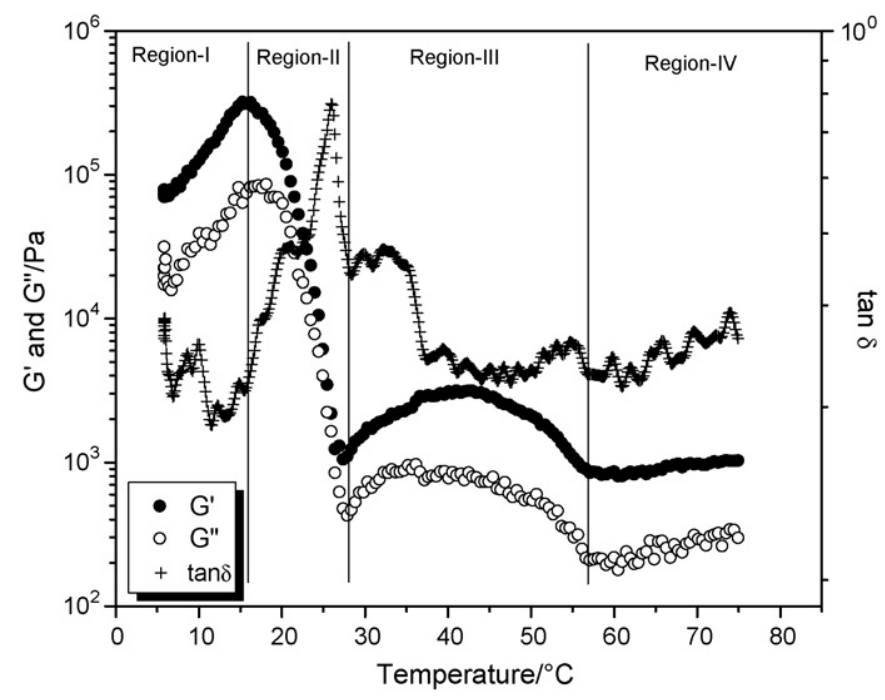

Fig. 9. Linear viscoelastic thermogram for $15 \mathrm{wt} \% \mathrm{NaO}$ mixture. Temperature scan of storage modulus $G^{\prime}$ (filled symbols), loss modulus $G^{\prime \prime}$ (open symbols) and loss tangent $G^{\prime \prime} / G^{\prime}$ (crosses) obtained by a time-cure test at $2 \%$ strain and $f=1 \mathrm{~Hz}$. Heating rate of the experiment was of $0.2^{\circ} \mathrm{C} / \mathrm{m}$ and the equilibration time of $2 \mathrm{~s}$.

like behaviour and the equilibrium structure of this hexagonal phase by using the so called "weak gel model".

A representative temperature dependence of viscoelastic moduli of the $\mathrm{H}_{1}$-phase of our surfactant system is that explored for the sample $c=18 \mathrm{wt} . \% \mathrm{NaO}$ and reported in Fig. 9. Previous strain-sweep experiments (here not reported) revealed that this hexagonal phase loses the linear viscoelastic response at $0.5 \%$ strain measuring at a frequency of $1 \mathrm{~Hz}$. On the other hand, this value is similar to that reported for the direct hexagonal phase of the sodium taurodeoxycholate/ $\mathrm{D}_{2} \mathrm{O}$ system [22]. The evolution of material functions of Fig. 9 reveals four different regions as the temperature was raised from 5 to $70^{\circ} \mathrm{C}$ :

- Region I, from 5 to $15^{\circ} \mathrm{C}$, shows a weak increase of both mechanical moduli. This region is characterized by low values of $\tan \delta$ revealing a strong gel-like behaviour. The rheological responses observed are in agreement with the presence of a two phase in equilibrium $\left(S_{\mathrm{h}}+W\right)$.

- Region II, which takes place above $15^{\circ} \mathrm{C}$, is characterized by a dramatic decrease in $G^{\prime}$ and $G^{\prime \prime}$ whereas $\tan \delta$ goes trough a pronounced maximum which is centred at $25^{\circ} \mathrm{C}$. Like DSC peaks observed in calorimetric thermograms, the loss tangent indicates the onset of the structural transition $\left(S_{\mathrm{h}}+W\right) \rightarrow \mathrm{H}_{1}$.

- Region III, which appears at temperature higher than $27^{\circ} \mathrm{C}$, corresponds to the direct hexagonal phase temperature interval. We observe again a low value of $\tan \delta$ while the mechanical moduli show an atypical change with the temperature. Up to $45^{\circ} \mathrm{C}$ both moduli increase, while they decrease with a further temperature rise as a consequence of $\mathrm{H}_{1} \rightarrow \mathrm{L}_{1}$ transition.

- Region $I V$, which takes place above $55^{\circ} \mathrm{C}$, shows a monotonic increase of both moduli as well as of $\tan \delta$. The liquid crystalline phase is completely melted and a viscoelastic solution appeared. 
The solution microstructure formed at temperatures $>57^{\circ} \mathrm{C}$ can be considered certainly different from that probed in more diluted isotropic mixtures (e.g. $12 \mathrm{wt} . \%$ at temperature above $30{ }^{\circ} \mathrm{C}$ ). The present experimental results indicate that gel-like behaviour recorded at high concentration is attributable to a micellar phase, with a structure consisting of cylinders that locally preserve an hexagonal-like order.

\subsection{The "weak-gel" model}

In binary and ternary phase diagrams of surfactant aqueous mixtures exist many single-phase regions with a gel-like character. While the gels have widely macroscopic structures they have common rheological properties. For the same materials, linear or quasi-linear viscoelastic measurements often reveal storage and loss modulus in nearly constant ratio, with the frequency dependence that is either a weak-power law or negligible. This behaviour persist down to the lowest accessible frequency range (about $10^{-3}-1 \mathrm{~Hz}$ depending on the system), in apparent contradiction to linear response theory which requires that $G^{\prime \prime}(\omega)$ should be an odd function of $\omega$. This behaviour could in principle be due to slow relaxation modes below the accessible frequency range.

This section concerns with an indirect analysis of microstructures and regards both concentrated micellar phase (sample at $c=15 \mathrm{wt} . \% \mathrm{NaO}$ ) or hexagonal liquid crystals. Linear viscoelastic spectra, discussed in previous figures, are now re-interpreted in accordance with the viscoelastic theory of Bohlin [29] and Winter [30]. This theory is reported in the literature as the "weakgel" model [31]. This model considers a "weak-gel" material as a flowing system characterized by weak physical interactions that cooperatively ensure the stability of the structure. The real structure of this material is made by a cooperative arrangement of flow units to form a strand. The weak-gel model provides a direct link between the microstructure of the material and its rheological properties. The most important parameter introduced in this model is the "coordination number", $z$, which corresponds to the number of flow units interacting with each other to give the observed flow response. According to the theory of the "weak-gel" model, the magnitude of complex modulus, $\left|G^{*}\right|=\left(G^{\prime 2}+G^{\prime \prime 2}\right)^{1 / 2}$, is expressed by [30]:

$\left|G^{*}(\omega)\right|=\mathrm{A} \omega^{1 / z}$

where $A$ is a proper constant which can be interpreted as the "interaction strength" between the rheological units, a sort of amplitude of cooperative interactions. Clearly, $\left|G^{*}\right| \log -\log$ plots versus $\omega$ should yield a straight line with slope $1 / z$ and intercept $A$.

Experimental values of $\left|G^{*}\right|$ for the mixtures analyzed in this work showed a good fit to Eq. (9) and therefore permitted an accurate estimate of $z$ and $A$. Fig. 10 shows the temperature dependence of $z$ and $A$ for the mixture $c=15 \mathrm{wt} . \% \mathrm{NaO}$. This sample presents a flow coordination number, $z$, close to unity in the temperature interval $30-45^{\circ} \mathrm{C}$ within the $\mathrm{L}_{1}^{*}$-area. At temperatures higher than $45^{\circ} \mathrm{C}$ (that is temperature at which the $\mathrm{L}_{1}^{*} \rightarrow \mathrm{L}_{1}$ phase transition occurs) $z$ suddenly increases and
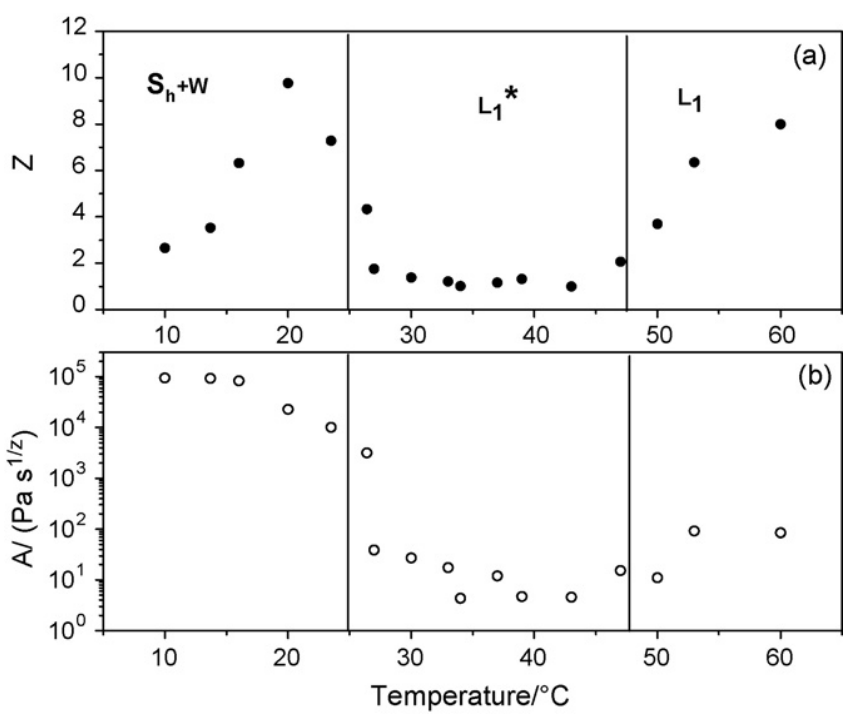

Fig. 10. Temperature evolution of "weak-gel" parameters for $15 \mathrm{wt} \% \mathrm{NaO}$ mixture: (a) the flow coordination number, $z$, and (b) the gel strength, A. Analysis along the phase sequences: $\left(S_{\mathrm{h}}+W\right) \rightarrow \mathrm{L}_{1}^{*} \rightarrow \mathrm{L}_{1}$.

reaches values of $z$ of ca. 6 . The $A$ profile in temperature follows a similar trend marking evident a structural change at two temperatures: the temperature of $26^{\circ} \mathrm{C}$ (transition from $S_{\mathrm{h}}+W$ to $\mathrm{L}_{1}$-phase) and the temperature of ca. $50^{\circ} \mathrm{C}$ (transition from $\mathrm{L}_{1}^{*}$ to $\mathrm{L}_{1}$-phase). In addition the formation of the $\mathrm{L}_{1}$-phase is accompanied by the presence of a strong elasticity. The values of $z$ measured within the $\mathrm{L}_{1}$-phase are in agreement with the average values found on hexagonal liquid-crystalline phases of some surfactant systems and well described by Bohlin [32]. As consequence the $\mathrm{L}_{1}$-phase of the $15 \mathrm{wt}$. $\%$ mixture evidences a six-fold coordination number, which can be attributed to a basic hexagonal microstructure consisting of long cylindrical micelles.

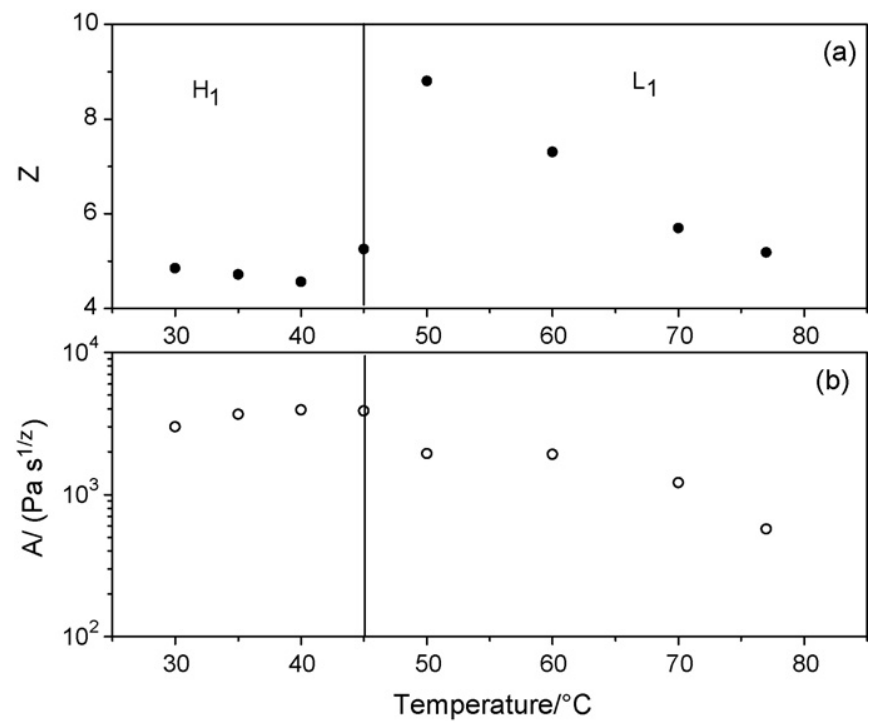

Fig. 11. Temperature evolution of "weak-gel" structural parameters for $18 \mathrm{wt} . \%$ $\mathrm{NaO}$ mixture: (a) the flow coordination number, $z$, and (b) the gel strength, $A$. Analysis along the phase sequence: $\mathrm{H}_{1} \rightarrow \mathrm{L}_{1}$. 
The temperature dependence of $z$ and $A$ for the mixture $c=18 \mathrm{wt} . \%$ is reported in Fig. 11. Both parameters appear to be almost constant with increasing temperature within the hexagonal region $\left(30-50^{\circ} \mathrm{C}\right)$ and evidence no structural changes of this phase by increasing the temperature. On the other hand, optical polarizing microscopy confirmed that hexagonal texture, of a fan-like type, is not modified during a temperature scan. In Fig. 10 it is also observed that $z$ values abruptly rise at the temperature of $55^{\circ} \mathrm{C}$ which correspond to hexagonal $\rightarrow$ micellar phase transition. It is worthy to point out that the values of $\mathrm{z}$ in the micellar phase are more or less comparable with cooperative coordination number of hexagonal phase found by Bohlin [32]. This fact suggests that the $\mathrm{L}_{1}$ structure of the $18 \mathrm{wt} . \%$ mixture at $T>50{ }^{\circ} \mathrm{C}$ is formed by cylindrical aggregates showing a highly local hexagonal order which disappears after a further temperature increase.

\section{Concluding remarks}

This contribution concerned with a detailed rheological investigation of binary mixtures of the $\mathrm{NaO} / \mathrm{H}_{2} \mathrm{O}$ system. We re-examined the phase diagram and the microstructures of $\mathrm{NaO}$ /water mixtures, from dilute up to the liquid crystalline region (hexagonal phase). Steady shear and linear viscoelastic experiments were performed in a wide composition and temperature interval for determining the relationship between the aggregate morphology and rheological properties. The analysis of data has followed both a qualitative and quantitative treatment.

Concerning the phase behaviour of the $\mathrm{NaO}$ /water system we observed that from the cmc (about $0.008 \mathrm{wt} . \%$ ) up to $10 \mathrm{wt} . \%$ the solutions contain small spherical micelles. Above this concentration the micelles start to grow and become anisometric in shape (cylinders). This rapid micellar growth (spheres $\rightarrow$ cylinders $\rightarrow$ long cylinders) is essentially due to the screening of electrostatic interaction with increasing surfactant composition. As a consequence, above $c=18 \mathrm{wt} . \% \mathrm{NaO}$, an hexagonal liquid crystal is formed. This phase consists of long cylindrical aggregates packed in a two-dimensional hexagonal array.

The rheological findings agreed very well with the phase studies. At low surfactant concentrations $(<10 \mathrm{wt} . \%)$ micelles appear as spherical aggregates. By a further increasing of concentration, $\mathrm{NaO}$ forms cylindrical aggregates with a rapid and sensitive uniaxial growth. The tail of dynamic moduli and their low values indicated the presence cylindrical micelles not entangled. An anomalous behaviour was observed for the mixture at $c=15 \mathrm{wt} . \% \mathrm{NaO}$, where a liquid-like and a gel-like response were observed at low and high temperatures, respectively. The liquid-like solution showed also a birefringence induced by stirring. The gel-like solution revealed the presence of cylindrical aggregates characterized by a local hexagonal order.

Finally, the $\mathrm{H}_{1}$ phase of the $\mathrm{NaO}$ /water system, which is polycrystalline, is characterized by a marked gel-like rheological response. Despite of common rheological data reported by hexagonal microstructures, the $\mathrm{H}_{1}$-phase of this system showed an highly ordered state and was able to sustain long-time stimuli.

\section{Acknowledgments}

The authors thank the "Ministero Italiano dell'Istruzione, dell'Universita' e della Ricerca " (PRIN_2003) to have partially supported this project.

FEA acknowledges Fundação para Ciência e Tecnologia (FCT) for a research grant (ref. SFRH/BD/8168/2002). The $\mathrm{PhD}$ student in our group, Adelino Abreu, is acknowledged for technical assistance.

\section{References}

[1] R. Murray, P. Mayes, V. Rodwell, D. Granner, Harper's Biochemistry, 26th ed., McGraw-Hill Companies, New York, 2003.

[2] B. Lindman, H. Wennerstrom, Phys. Rep. (1979) 1-86.

[3] G.J.T. Tiddy, Phys. Rep. (1980) 1-65.

[4] U. Tepeil, L. Heymann, N. Aksel, Colloids Surf. A 193 (2001) 35.

[5] H. Rehage, H. Hoffmann, Mol. Phys. 74 (1991) 933.

[6] C. Rodriquez-Abreu, D.P. Acharya, K. Aramaki, H. Kunieda, Colloid Surf. A 269 (2005) 59;

D.P. Acharya, H. Kunieda, Phys. Chem. B 107 (2003) 10188.

[7] J. Bornè, T. Nylander, A. Khan, Langmuir 17 (2001) 7742.

[8] J. Bornè, PhD Thesis Lund University, Lund (Sweden), 2002.

[9] F.E. Antunes, PhD Thesis, Coimbra University (Portugal), 2006.

[10] R.D. Vold, J. Chem. 43 (1939) 1213

[11] P. Luzzati, H. Mustacchi, A. Skoulios, F. Husson, Acta Cryst. 13 (1960) 660.

[12] J.D. Ferry, Viscoelastic Properties of Polymers, 3rd ed., Wiley, New York, 1980.

[13] F.A. Morrison, Understanding Rheology, Oxford University Press, Oxford, 2001.

[14] M.R. Mackley, R.T.J. Marshall, J.B.A.F. Smeulders, Chem. Eng. Sci. 49 (1994) 2551.

[15] E.O. Stejskal, J.E. Tanner, J. Phys. Chem. 42 (1965) 288.

[16] N. Mahieu, D. Canet, J.M. Cases, J.C. Boubel, J. Phys. Chem. 95 (1991) 1844 ;

K. Edwards, M. Silvander, G. Karlsson, Langmuir 11 (1995) 2429.

[17] C.W. Macosko, Rheology: Principles, Measurements and Applications, Wiley-VCH, New York, 1993.

[18] T. Shikata, H. Hirata, T. Kotaka, Langmuir 3 (1987) 1081; T. Shikata, H. Hirata, T. Kotaka, Langmuir 4 (1988) 1081.

[19] H. Rheage, H. Hoffmann, J. Phys. Chem. 92 (1988) 4712.

[20] J.F.A. Soltero, J.E. Puig, O. Manero, P.C. Schulz, Langmuir 11 (1995) 3337.

[21] L. Coppola, C. Oliviero, U. Olsson, G.A. Ranieri, Langmuir 16 (2000) 4180.

[22] L. Coppola, R. Gianferri, C. Oliviero, I. Nicotera, Langmuir 19 (2003) 1990.

[23] L. Coppola, C. Oliviero, L. Pogliani, G.A. Ranieri, M. Terenzi, Colloid Polym. Sci. 278 (2000) 434.

[24] R. Lapasin, S. Pricl, Rheology of Industrial Polysaccharides: Theory and Application, Blackie Academic Professional, London, 1995.

[25] M. Mours, H.H. Winter, Mechanical spectroscopy, in: T. Tanaka (Ed.), On "Experimental methods in Polymer Science", Academic Press, San Diego, 2000.

[26] R. Mezzenga, C. Meyer, C. Servais, A.I. Romanescu, L. Sagalowicz, R.C. Hayward, Langmuir 21 (2005) 3322.

[27] G. Montalvo, M. Valiente, E. Rodenas, Langmuir 12 (1996) 5202.

[28] L. Coppola, R. Gianferri, C. Oliviero, G.A. Ranieri, J. Colloid Interf. Sci. 264 (2003) 554.

[29] L. Bohlin, J. Colloid Interf. Sci. 74 (1980) 423.

[30] H.H. Winter, Polym. Eng. Sci. 27 (1987) 1698.

[31] D. Gabriele, B. de Cindio, P. D’Antona, Rheol. Acta 40 (2001) 120.

[32] L. Bohlin, J. Colloid Interf. Sci. 69 (1979) 194. 